\title{
Increased fibrinolysis-induced bradykinin formation in hereditary angioedema confirmed using stored plasma and biotechnological inhibitors
}

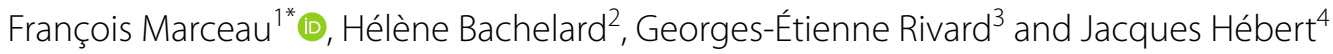

\begin{abstract}
Objective: We recently investigated the pathways of immunoreactive bradykinin (iBK) formation in fresh blood of normal volunteers and of patients with hereditary angioedema due to C1-esterase inhibitor deficiency (HAE-1/-2). Herein, we adapted the techniques to small volumes $(200 \mu \mathrm{l})$ of previously frozen citrated plasma and further analyzed the mechanisms of iBK formation with additional biotechnological inhibitors.

Results: Measurable iBK formation was observed under stimulation with tissue kallikrein (KLK-1, $10 \mathrm{nM})$, the particulate material Kontact-APTT (concentration reduced to $2 \% \mathrm{v} / \mathrm{v}$ ) or recombinant tissue plasminogen activator (tPA, 169 nM), with little background in unstimulated plasma incubated for up to $2 \mathrm{~h}$. Plasma samples from HAE-1/-2 patients responded earlier to TPA than those from controls, as previously reported with whole blood. Lanadelumab inhibited iBK formation induced by Kontact-APTT and tPA. A highly specific plasmin inhibitor, DX-1000, abolished tPA-induced iBK formation in plasma but had no effect against Kontact-APTT, confirming the role of fibrinolysis in tPA-induced kinin formation. The anti-lanadelumab neutralizing antibody M293-D02 reversed the inhibitory effects of lanadelumab. Frozen plasma is a suitable material for measuring iBK formation kinetics, with possible applications such as investigating the effect of rare disease states on the kallikrein-kinin system and monitoring the effect of HAE prophylactic treatments.
\end{abstract}

Keywords: Bradykinin, Hereditary angioedema with C1-INH deficiency, Kallikreins, Fibrinolysis, Lanadelumab

\section{Introduction}

We recently investigated the pathways of immunoreactive bradykinin (iBK) formation in fresh blood of normal volunteers and of patients with hereditary angioedema (HAE) due to $\mathrm{C} 1$ esterase inhibitor (C1-INH) deficiency (mutations of the SERPING1 gene) [1]. In the case of type 1 and 2 HAE (HAE-1, HAE-2) patients, the blood was sampled during remission. Blood samples were submitted to various standardized forms of in vitro stimulation before extraction. It was found that contact system activation, recombinant tissue kallikrein (KLK-1) or

\footnotetext{
*Correspondence: francois.marceau@crchudequebec.ulaval.ca

${ }^{1}$ Axe Microbiologie-Infectiologie et Immunologie, CHU de QuébecUniversité Laval, Quebec, QC G1V 4G2, Canada

Full list of author information is available at the end of the article
}

tissue plasminogen activator (tPA) triggered abundant iBK formation, but not the activation of platelets or leukocytes present in the fresh blood. Only tPA was significantly more active in HAE patients, releasing iBK faster and more intensely during the 1 st $h$ of incubation [1].

The present work has several goals. Since blood cells do not contribute to kinin formation in a measurable manner, the platform could be more versatile by testing iBK formation in citrated plasma using a scaled down sample volume (200 $\mu \mathrm{l}$ per experimental point) and light benchtop equipment. Also, we decreased the intensity of the activation of contact system with KontactAPTT to detect possible differences between healthy volunteers and HAE patients. Remaining frozen plasma samples from subsets of previously studied healthy volunteers or HAE patients were exploited to validate these 
approaches. Additional biotechnological inhibitors, the plasmin inhibitor DX-1000 [2] and the plasma kallikrein active site blocking monoclonal antibody, lanadelumab (DX-2930) [3] were used to analyze the mechanisms of iBK formation triggered by selected stimuli. Lanadelumab and other plasma kallikrein inhibitors are being clinically deployed for prophylaxis of HAE attacks [4-6]: our platform may be applied to monitor the effect of the administration of such inhibitors in HAE patients. Characterizing iBK formation in the genetically heterogeneous HAE patients/families with normal C1-INH levels is also an interesting future goal [7]. Indeed, recently discovered mutations of the F12 and PLG genes [8, 9] beg for a physiopathological analysis of the kallikrein-kinin system. Because HAE caused by a mutation in a gene different from SERPING1 is very rare, updated techniques based on easily shipped frozen plasma will make possible a global recruitment of these patients for the physiopathological analysis of iBK formation.

\section{Main text \\ Materials and methods Human participants}

This research project has been approved by the ethics board Comité d'éthique de la recherche, CHU de QuébecUniversité Laval, file 2018-3857. Adult healthy human subjects or unrelated HAE-1 or -2 patients were studied, during a remission period for the patients. Subject characteristics are described in Additional file 1: Table S1. Venous blood anticoagulated with sodium citrate was obtained without contact with glass [1]. Both volunteers and patients used in the present study constitute subsets of subjects included in the previous study [1], as the remaining fresh blood had been centrifuged, their plasma frozen on the day of their blood sampling and kept at $-80{ }^{\circ} \mathrm{C}$ since then. HAE-1/-2 diagnosis was supported by the measurement of $\mathrm{C} 4$ and $\mathrm{C} 1-\mathrm{INH}$ [1] (data reported in Additional file 1: Table S1).

\section{Enzyme immunoassay (EIA) of BK}

Aliquoted frozen plasma from the previous study [1] was used in all experiment. Each iBK concentration value was derived from $200 \mu \mathrm{l}$ of thawed citrated plasma transferred to a $1.5 \mathrm{ml}$ Eppendorf conical test tube. Table 1 lists stimulatory and inhibitory substances that have been added to plasma to probe the pathways of iBK formation. All tubes contained the angiotensin converting enzyme (ACE) inhibitor enalaprilat (final concentration $130 \mathrm{nM}$ ) to isolate the formation of $\mathrm{BK}$ from its rapid hydrolysis by its most important inactivating enzyme $[10,11]$. The final concentration of KLK-1 (10 nM) and of tPA (169 nM) used in the present experiments have been used previously with whole blood [1] but represent relatively weaker stimuli in the present study because the recombinant proteins were excluded from the blood volume occupied by blood cells. The concentration of Kontact-APTT (2\%) has been greatly decreased vs. the previous study involving fresh blood $(20 \% \mathrm{v} / \mathrm{v})$. The tubes were incubated under rotary agitation (300 rpm) in a pre-equilibrated $\left(37^{\circ} \mathrm{C}\right)$ Thermomixer Compact apparatus with $1.5 \mathrm{ml}$ block (Eppendorf) for 0-120 min. When the desired incubation duration was reached, $1 \mathrm{ml}$ of cold $\left(-20{ }^{\circ} \mathrm{C}\right)$ absolute ethanol was added to each tube to precipitate/denature proteins. Tubes were allowed to sit for at least $1 \mathrm{~h}$ on ice, and then were centrifuged $(13,000 \mathrm{~g}$, $1 \mathrm{~min}$, room temperature, Microfuge 16, Beckman Coulter). The translucent supernatants were transferred in a new set of tubes and completely dried (SpeedVac concentrator). The dried extracts, kept at $-80{ }^{\circ} \mathrm{C}$, were resuspended in $200 \mu \mathrm{H} \mathrm{H}_{2} \mathrm{O}$ for iBK determination. The BK enzyme immunoassay (EIA) kits (Phoenix Pharmaceuticals, Burlingame, CA) contain an assay buffer that was used to further dilute the reconstituted samples (100- or

Table 1 Stimuli, potentiator or inhibitors of iBK formation

\begin{tabular}{|c|c|c|c|}
\hline Stimulus & $\begin{array}{l}\text { Final } \\
\text { concentration } \\
\text { in plasma }\end{array}$ & Site of action & Source \\
\hline Recombinant active KLK-1 & $10 \mathrm{nM}$ & Kininogen cleavage, mostly low molecular weight form & DiaMedica, Inc. \\
\hline Pacific Hemostasis Kontact-APTT & $\begin{array}{l}2 \% \text { v/v without } \\
\text { the calcium } \\
\text { supplement }\end{array}$ & Particulate material that triggers the contact system & ThermoFisher Scientific \\
\hline Recombinant tPA (alteplase, Cathflow) & $169 \mathrm{nM}$ & Plasminogen activation & Roche \\
\hline Enalaprilat & $130 \mathrm{nM}$ & ACE inhibition & Kemprotec Ltd. (Maltby, UK) \\
\hline $\begin{array}{l}\text { Lanadelumab (DX-2930), humanized mono- } \\
\text { clonal antibody }\end{array}$ & $500 \mathrm{nM}$ & Inhibitor of plasma kallikrein [3] & Shire Intl. GmbH \\
\hline M293-D02, monoclonal antibody & $1 \mu \mathrm{M}$ & Anti-lanadelumab neutralizing antibody [15] & Shire Intl. GmbH \\
\hline DX-1000, recombinant Kunitz-type inhibitor & $1 \mu \mathrm{M}$ & Inhibitor of plasmin [2] & Shire Intl. GmbH \\
\hline
\end{tabular}


1000-fold) before direct application to the EIA, used as directed (duplicate determination) [1].

\section{Data analysis}

Values are mean \pm standard error of the mean (S.E.M.). In the first part of the study (iBK kinetics as a function of time), single comparisons of value pairs were performed with Mann-Whitney test since the variances significantly differed (Prism 5.0, GraphPad Software Inc., San Diego, CA). In the study of inhibitors, sets of values were compared with the Kruskal-Wallis test (non-parametric ANOVA) followed by Dunn's multiple comparison test to compare selected pairs of values.

\section{Results and discussion}

\section{Effect of established stimulants of iBK formation on samples} of blood plasma

For the first part of the study, the previously frozen plasma from a subset of subjects enrolled in the previous project was submitted to in vitro activation (7 controls and 9 HAE patients; human subjects described in Additional file 1: Table S1). In all reported experiments, the ACE inhibitor enalaprilat was present to reduce iBK breakdown and isolate the kinetics of kinin formation in a more discriminating manner, as previously shown [1]. As reported for whole blood samples [1], iBK remained at background concentrations $(\leq 0.4 \mathrm{ng} / \mathrm{ml})$ in control plasma maintained for $0-2 \mathrm{~h}$ at $37^{\circ} \mathrm{C}$, whether the samples were from healthy subjects or HAE patients (Fig. 1). Again consistent with findings based on whole blood, iBK concentrations were quickly and persistently increased in response to active KLK-1 (10 $\mathrm{nM})$ in plasma from either type of subjects, with a slow decline in the presence of enalaprilat. The particulate material Kontact-APTT, used as an activator of the contact system, has been added here in plasma in a much diluted manner (final concentration $2 \% \mathrm{v} / \mathrm{v})$ relative to the concentration previously used in whole blood $(20 \% \mathrm{v} / \mathrm{v})$. However, iBK kinetics remained

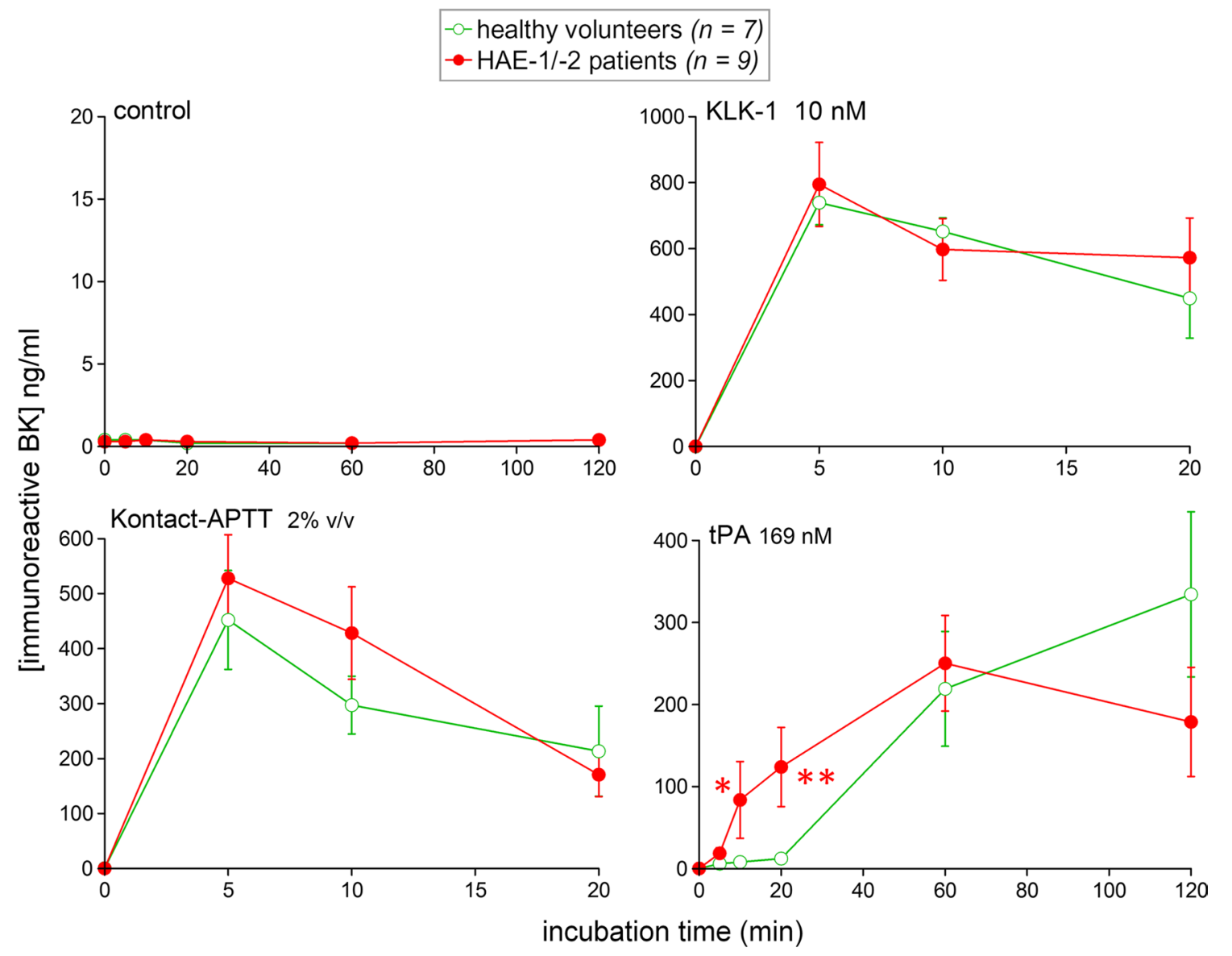

Fig. 1 Variation of iBK concentrations as a function of time and stimulation in samples of previously stored citrated blood plasma (incubation at $37^{\circ} \mathrm{C}$ in the presence of enalaprilat, $130 \mathrm{nM}$, added at time zero). Plasma samples originated from healthy subjects or HAE patients with C1-INH deficiency. Values are mean \pm S.E.M. for a number of subjects indicated by $n$. For each experimental condition and time point, Mann-Whitney test was applied to isolate the effect of the disease $\left({ }^{*} \mathrm{P}<0.01 ;{ }^{*} \mathrm{P}<0.001\right)$ 
similar in amplitude vs. those of the previous study, with again no significant effect of the disease (Fig. 1).

tPA retained its very slow kinetics of iBK formation in plasma from healthy volunteers, the first accumulation taking place after 60 min of incubation (Fig. 1). In plasma from HAE patients, a much earlier ( $\geq 10 \mathrm{~min}$ ) and initially stronger response was seen, as reported with whole blood [1]. At the time points 10 and $20 \mathrm{~min}$, the disease had a highly significant effect.

\section{Effect of novel biotechnological inhibitors}

In the previous study based on blood [1], the formation of iBK induced by tPA or Kontact-APTT was abolished by biotechnological inhibitors of plasma kallikrein, either the Kunitz-type peptide inhibitor EPICAL2 [12] or the humanized monoclonal antibody M202-H03 [3]. tPA was further inhibited by the active site inhibitor of factor XII, the monoclonal antibody DX-4012 [13]. KLK1 -induced iBK formation was unaffected by any of these inhibitors, but was virtually abolished by the active sitedirected specific inhibitor of that protease, the monoclonal DX-2300 [14].

Additional inhibitors were combined with two activators of iBK formation: the highly selective Kunitztype plasmin inhibitor, DX-1000 [2] and lanadelumab, a plasma kallikrein inhibitor recently introduced in clinical use for the prevention of HAE attacks $[4,15]$. Biotechnological inhibitors were added to plasma samples from 7 or 8 healthy volunteers $5 \mathrm{~min}$ before one of the 2 active stimuli was added. The incubation periods selected for Kontact-APTT and tPA were those that produced maximal iBK concentrations (5 and $60 \mathrm{~min}$, respectively) and stimulus concentrations were the same as in the first part of the study (Fig. 1); enalaprilat presence was maintained as well (130 nM). Only lanadelumab significantly reduced iBK formation induced by Kontact-APTT (Fig. 2). Further, the anti-lanadelumab antibody M292-D02 [14], without effect by itself, significantly reversed the inhibition caused by lanadelumab. The same findings applied to $\mathrm{tPA}$-induced iBK formation, except that the plasmin inhibitor DX-1000, without effect against Kontact-APTT, was inhibitory against tPA (Fig. 2). The results confirm that tPA recruits an intermediate step, the activation of plasminogen, to trigger the contact system, probably via factor XII cleavage as previously discussed [1]. The measurement of iBK formation as a function of time may be suitable to investigate the effect of plasma kallikrein inhibitors in the plasma of HAE patients, especially if blood is also sampled before the initiation of the prophylactic treatment for baseline comparison. As the anti-lanadelumab antibody M292-D02 was confirmed to reverse in vitro the inhibitory effect of lanadelumab on iBK formation, M293-D02 could be an additional tool



in the monitoring of lanadelumab prophylactic treatment. The platform may be suitable to study the role of $\mathrm{BK}$ in other clinical conditions such as HAE with normal C1-INH or acquired forms of angioedema.

Thus, HAE-associated changes in iBK formation were confirmed under the modified experimental conditions based on stored and frozen plasma: hypersensitivity to tPA remains the essential change, with no significant difference concerning direct contact system activation in the plasma of HAE- 1 or -2 patients. This suggests that the instability of the kallikrein-kinin system in HAE-1/-2 resides in the upstream fibrinolytic system, as previously 
discussed [1]. Known triggers of HAE attacks include plausible situations where fibrinolysis may be activated: trauma, infection, medical and dental procedures, menstruation, and, singularly, mental stress [16, 17]. Indeed, mental stress determines tPA release and fibrin turnover in healthy human subjects [18].

\section{Limitations}

In general, the kinetics of iBK formation under the three types of stimulation provide a fully quantitative and rich source of information relative to other methods proposed to study the kallikrein-kinin system in HAE patients, such as the consumption of high molecular weight kininogen with detection of cleavage products that may be based on immunoblotting, a semi-quantitative approach. We have not sampled HAE patients during an attack. Further, most patients received a prophylactic treatment of plasma-derived C1-INH (Berinert), which was not interrupted for the study; any effect on blood chemistry was minimized by sampling the blood at the end of their administration cycle. In fact, the C1-INH levels and $\mathrm{C} 4$ consumption were not normalized in the blood of patients under Berinert prophylaxis (Additional file 1: Table S1). The previous study included the corroboration of the presence of a BK-like agonist in extracts of blood samples based on signaling of cultured cells expressing the recombinant $B_{2}$ receptor [1]; this verification was not extended to the present study. KLK-1 is not a part of the contact system and its effect on iBK generation is not modified by HAE- $1 /-2$, but its role is not excluded in other forms of angioedema or anaphylactoid states.

\section{Additional file}

Additional file 1: Table S1. Characteristics of human subjects in experiments reported in Fig. 1: patients with HAE with C1-INH deficiency (HAE-1, HAE-2; 6 females, 3 males) or healthy volunteers (5 females, 2 males). For each human subject: age range, diagnosis, approximate frequency of attacks, prophylactic treatment, blood levels of $\mathrm{C} 4$ and $\mathrm{C} 1-\mathrm{NNH}$.

\section{Abbreviations}

ACE: angiotensin converting enzyme; BK: bradykinin; HAE: hereditary angioedema; HAE-1: type 1 HAE; HAE-2: type $2 \mathrm{HAE}$; iBK: immunoreactive bradykinin; KLK-1: tissue kallikrein; tPA: tissue plasminogen activator.

\section{Acknowledgements \\ Dr. Daniel J. Sexton (Shire, now part of the Takeda group of companies) provided the protease inhibitors and usefully discussed the experimental approaches and results. Technical help from Ms. Johanne Bouthillier and the gift of KLK-1 from DiaMedica, Inc. (Minneapolis, MN, USA) are gratefully acknowledged.}

\section{Authors' contributions}

$F M, H B$, GER and JH designed the experiments; FM and $H B$ participated to experimental work. FM analyzed results and wrote the manuscript. All authors read and approved the final manuscript.

\section{Funding}

Supported by the Investigator-Initiated Research contract IIR-CAN-001615 from Shire International $\mathrm{GmbH}$ (now part of the Takeda group of companies). The sponsor had no role in the design of the study and collection, analysis, and interpretation of data and writing of the manuscript.

\section{Availability of data and materials}

The datasets used in the present study are available from the corresponding author on reasonable request. The biotechnological inhibitors were provided by Shire (now part of the Takeda group of companies) and KLK-1, from DiaMedica Therapeutics. All other reagents are commercially available.

\section{Ethics approval and consent to participate}

The local ethical review board (Comité d'éthique de la recherche, $\mathrm{CHU}$ de Québec-Université Laval) granted ethical approval to carry out the study involving blood donations (file no. 2018-3857). All study subjects gave written informed consent.

\section{Consent for publication}

Not applicable.

\section{Competing interests}

FM. served as a consultant and received research funds from for Pharvaris B.V., outside the submitted work. G.E.R. has been a member of advisory boards (Baxalta, Bayer, Biogen Idec, CSL Berhing, Novo Nordisk, Octapharma, Pfizer) and received funding from Bayer, CSL Behring and Pfizer (unrelated to the submitted work). J.H. has been a speaker/teacher for CLS Behring, Novartis, Shire and Aralez; he has been a member of advisory committees (AstraZeneca, CLS Behring, Shire, and Novartis) and a clinical investigator for Circassia, Merck (ALK), Stallergene, Boehringer-Ingelheim, GlaxoSmithKline (GSK), Teva, Novartis, Sanofi, AstraZeneca, Johnson \& Johnson, CLS Behring, Shire, Roche, Green Cross and Griffols (unrelated to the submitted work).

\section{Author details}

${ }_{1}^{1}$ Axe Microbiologie-Infectiologie et Immunologie, CHU de Québec-Université Laval, Quebec, QC G1V 4G2, Canada. ${ }^{2}$ Axe Endocrinologie et Néphrologie, CHU de Québec-Université Laval, Quebec, QC G1V 4G2, Canada. ${ }^{3}$ Division of Hematology/Oncology, CHU Ste-Justine, Montreal, QC H3T 1C5, Canada. ${ }^{4}$ Service d'allergie, CHU de Québec-Université Laval, Quebec, QC G1V 4G2, Canada.

Received: 7 March 2019 Accepted: 22 May 2019

Published online: 27 May 2019

\section{References}

1. Charest-Morin X, Hébert J, Rivard GÉ, Bonnefoy A, Wagner É, Marceau F. Comparing pathways of bradykinin formation in whole blood from healthy volunteers and patients with hereditary angioedema due to $\mathrm{C} 1$ inhibitor deficiency. Front Immunol. 2018;9:2183.

2. Devy L, Rabbani SA, Stochl M, Ruskowski M, Mackie I, Naa L, et al. PEGylated DX-1000: pharmacokinetics and antineoplastic activity of a specific plasmin inhibitor. Neoplasia. 2007;9(11):927-37.

3. Kenniston JA, Faucette RR, Martik D, Comeau SR, Lindberg AP, Kopacz KJ, et al. Inhibition of plasma kallikrein by a highly specific active site blocking antibody. J Biol Chem. 2014;289(34):23596-608.

4. Banerji A, Riedl MA, Bernstein JA, Cicardi M, Longhurst HJ, Zuraw BL, et al. Effect of lanadelumab compared with placebo on prevention of hereditary angioedema attacks: a randomized clinical trial. JAMA. 2018;320(20):2108-21.

5. Aygören-Pürsün E, Bygum A, Grivcheva-Panovska V, Magerl M, Graff J, Steiner UC, et al. Oral plasma kallikrein inhibitor for prophylaxis in hereditary angioedema. N Engl J Med. 2018;379(4):352-62.

6. Feener EP, Murugesan N, Robson P, Li L, Rushbrooke L, Thoonen R, et al. KVD900 protects high molecular weight kininogen from ex vivo plasma kallikrein-mediated cleavage in plasma from patients with hereditary angioedema (HAE): results from capillary-based immunoassay. European Academy of Allergy and Clinical Immunology 2018 Congress, 26-30 May 2018, Munich, Germany. https://www.kalvista.com/sites/default/files /2018-05/EAACI2018.pdf. Accessed 23 May 2019. 
7. Lara-Marquez ML, Christiansen SC, Riedl MA, Herschbach J, Zuraw BL. Threshold-stimulated kallikrein activity distinguishes bradykinin- from histamine-mediated angioedema. Clin Exp Allergy. 2018;48(11):1429-38.

8. de Maat S, Björkqvist J, Suffritti C, Wiesenekker CP, Nagtegaal W, Koekman $A$, et al. Plasmin is a natural trigger for bradykinin production in patients with hereditary angioedema with factor XII mutations. J Allergy Clin Immunol. 2016;138(5):1414-23.

9. Bork K, Wulff K, Steinmüller-Magin L, Braenne I, Staubach-Renz P, Witzke $\mathrm{G}$, et al. Hereditary angioedema with a mutation in the plasminogen gene. Allergy. 2018;73(2):442-50.

10. Cyr M, Lepage Y, Blais C, Gervais N, Cugno M, Rouleau JL, et al. Bradykinin and des-Arg ${ }^{9}$-bradykinin metabolic pathways and kinetics of activation of human plasma. Am J Physiol Heart Circul Physiol. 2001;281(1):H275-83.

11. Fryer RM, Segreti J, Banfor PN, Widomski DL, Backes BJ, Lin CW, et al. Effect of bradykinin metabolism inhibitors on evoked hypotension in rats: rank efficacy of enzymes associated with bradykinin-mediated angioedema. Br J Pharmacol. 2008;153(5):947-55.

12. Dai J, Agelan A, Yang A, Zuluaga V, Sexton D, Colman RW, et al. A role for plasma kallikrein-kinin system activation in the synovial recruitment of endothelial progenitor cells in arthritis. Arthritis Rheum. 2012;64(11):3574-82.

13. Kokoye Y, Ivanov I, Cheng Q, Matafonov A, Dickeson SK, Mason S, et al. A comparison of the effects of factor XII deficiency and prekallikrein deficiency on thrombus formation. Thromb Res. 2016;140:118-24.
14. Sexton DJ, Chen T, Martik D, Kuzmic P, Kuang G, Chen J, et al. Specific inhibition of tissue kallikrein 1 with a human monoclonal antibody reveals a potential role in airway diseases. Biochem J. 2009;422(2):383-92.

15. Banerji A, Busse P, Shennal M, Lumry W, Davis-Lorton M, Wedner HJ, et al. Inhibiting plasma kallikrein for hereditary angioedema prophylaxis. N Engl J Med. 2017;376(8):717-28.

16. Zotter Z, Csuka D, Szabó E, Czaller I, Nébenführer Z, Temesszentandrási G, et al. The influence of trigger factors on hereditary angioedema due to C1-inhibitor deficiency. Orphanet J Rare Dis. 2014:9:44

17. Saravese L, Bova M, De Falco R, Guarino MD, De Luca Picione R, Petraroli $A$, et al. Emotional processes and stress in children affected by hereditary angioedema with C1-inhibitor deficiency: a multicenter, prospective study. Orphanet J Rare Dis. 2018;13:115.

18. von Känel R. Acute mental stress and hemostasis: when physiology becomes vascular harm. Thromb Res. 2015;135(Suppl. 1):S52-5.

\section{Publisher's Note}

Springer Nature remains neutral with regard to jurisdictional claims in published maps and institutional affiliations.
Ready to submit your research? Choose BMC and benefit from:

- fast, convenient online submission

- thorough peer review by experienced researchers in your field

- rapid publication on acceptance

- support for research data, including large and complex data types

- gold Open Access which fosters wider collaboration and increased citations

- maximum visibility for your research: over 100M website views per year

At BMC, research is always in progress.

Learn more biomedcentral.com/submissions 